\title{
A New Test Method on the Convergence and Divergence for Infinite Integral
}

\author{
Guocheng Li \\ Linyi University at Feixian \\ Feixian, Shandong, P.R.China
}

\begin{abstract}
The way to distinguish convergence or divergence of an infinite integral on non-negative continuous function is the important and difficult question in the mathematical teaching all the time. Using the comparison of integrands to judge some exceptional infinite integrals are hard or even useless. In this paper, we establish the exponential integrating factor of negative function, and present a new method to test based on its exponential integrating factor. These conclusions are convenient and valid additions of the previously known results.
\end{abstract}

Keywords-Iinfinite integral; Exponential integrating factor; Convergence and divergence.

\section{INTRODUCTION}

The convergence and divergence of infinite integral plays a significant role in mathematical analysis, and has been received much attention of many researchers. Many effective methods have been proposed such as Ordinary Comparison Test, Limiting Test, Dirichlet Test and Abel Test [1]. The basic ideas of these methods are to find another comparison infinite integral that its convergence or divergence is certain. But it is difficult or impossible to find the comparison infinite. So these arouse great interest of many scholars to research ([2-3]).

It is well-known that the convergence and divergence of infinite integral for the different integrand which its limit is zero is different. Base on the geometric meaning of infinite integral, it equal to the size of the area which is surrounded by the integrand (image) and $\mathrm{X}$ axis (not closed). The Convergence and divergence mainly determined by the proximity degree of integrand tends to the $\mathrm{x}$-axis. In this paper, we discuss the test method for the convergence and divergence

of infinite integral $\int_{a}^{+\infty} f(x) d x \square$ where $f(x) \square$ is the nonnegative continuous function which is defined in the interval $[a,+\infty), a \geq 0 \square$ Although we have solved part of the problem by $\ln f(x) / \ln x \square$ in[3], it can't intuitive describe the proximity degree of integrand tends to the $\mathrm{x}$-axis, so the exponential integrating factor of negative function is created and we obtain a new test method for the convergence and divergence of infinite integral. This method is more simple and feasible than ever before.

\section{CHARACTERIZATIONS ON CONVERGENCE OF INFINITE INTEGRAL}

Definition 2.1 Assume that $f(x)$ is non-negative differentiable function, we call the formula

$R(x)=-f^{\prime}(x) / f(x)$ is the function of the exponential integrating factor of $f(x)$, abbreviated as factor function.

From definition 2.1, we suppose the function

$$
f(x)=f\left(x_{0}\right) \exp \left\{-\int_{x_{0}}^{x} R(t) d t\right\}, x_{0} \geq a \text { is completely }
$$

determined by its factor function $R(x)$. When the independent variable tend to be infinite, we can use the size of factor function to compare the different functions reduce speed of which tend to the $\mathrm{x}$-axis. For $\lim _{x \rightarrow+\infty} f(x)=0$ is the necessary condition of convergence for infinite integrals $\int_{a}^{+\infty} f(x) d x([1])$, so we can easy to push that $R(x) \geq 0$ is the necessary conditions of convergence for $\int_{a}^{+\infty} f(x) d x$.

If $\lim _{x \rightarrow+\infty} \frac{f(x)}{g(x)}=c>0$, we have $f(x)$ and $g(x)$ are equivalent. We denoted by $f \sim^{t} g$. The nature of the equivalent functions is incredibly similar, so what about the infinite integration convergence of the equivalent integrand? We have the following conclusion.

Theorem 2.1 Assume that Non-negative differentiable functions $f(x)$ and $g(x)$ are equivalent, then its convergence and divergence is incredibly similar.

Proof: By $\lim _{x \rightarrow \infty} \frac{\int_{a}^{x} f(t) d t}{\int_{a}^{x} g(t) d t}=\lim _{x \rightarrow \infty} \frac{f(x)}{g(x)}=c>0$, 
There exist constant $m, M>0$ such that

$m \leq f(x) / g(x) \leq M, \forall x>a$. Obviously, we have

$$
\begin{aligned}
& m \int_{a}^{+\infty} g(x) d x \leq \int_{a}^{+\infty} f(x) d x \leq M \int_{a}^{+\infty} g(x) d x ; \\
& \frac{1}{M} \int_{a}^{+\infty} f(x) d x \leq \int_{a}^{+\infty} g(x) d x \leq \frac{1}{m} \int_{a}^{+\infty} f(x) d x
\end{aligned}
$$

From the definition of convergence of the infinite integrals, the desired result follows.

Theorem 2.2 Assume that $f(x), g(x)$ are nonnegative differentiable functions in interval $[a,+\infty)$ and

$f(x) / g(x)$ is finally monotone decreasing, then

(i) $\exists x_{0}, \forall x>x_{0}, R_{f}(x)>R_{g}(x)$.

(ii) If $\int_{a}^{+\infty} g(x) d x$ is convergent, then $\int_{a}^{+\infty} f(x) d x$ is convergent, If $\int_{a}^{+\infty} g(x) d x$ is divergent, then $\int_{a}^{+\infty} f(x) d x$ is divergent.

Proof:

(i) $\exists x_{0}, \forall x>x_{0}$,

$$
\begin{aligned}
& \left(\frac{f(x)}{g(x)}\right)^{\prime}=\frac{g(x) f^{\prime}(x)-f(x) g^{\prime}(x)}{g^{2}(x)}= \\
& \frac{1}{f(x) g(x)}\left[R_{g}(x)-R_{f}(x)\right]<0 \\
& \Leftrightarrow R_{f}(x)>R_{g}(x) .
\end{aligned}
$$

(ii) $\exists M>0$, let $\frac{f(x)}{g(x)} \leq M$, then

$\int_{a}^{+\infty} f(x) d x \leq M \int_{a}^{+\infty} g(x) d x$. Using Ordinary Comparison

Test, we obtain this conclusion.

Assume that we know the convergence and divergence of an infinite integral, we can obtain the convergence and divergence of other infinite integrals by comparing the size of the exponential factor. But it's complex. In fact, it can be derived alone by the exponential factor $R(x)$ of integrand.

Theorem 2.3 Assume that $f(x)$ is non-negative differentiable function in $[a,+\infty)$,

(i) If $\lim _{x \rightarrow+\infty} x R(x)>1$, then $\int_{a}^{+\infty} f(x) d x$ is convergent, (ii) If $\lim _{x \rightarrow+\infty} x R(x)<1$, then $\int_{a}^{+\infty} f(x) d x$ is divergent.

Proof: (i) $\forall k>0$, we have $\frac{-f^{\prime}(x)}{f(x)} \geq \frac{1+k}{x}$, then

$$
-\ln f(x) \geq \ln \left(c x^{1+k}\right), c>0 .
$$

That is $f(x) \leq \frac{1}{c} x^{-(1+k)}$ i.e., According to the Ordinary Comparison Test, $\int_{a}^{+\infty} f(x) d x$ is convergent.

(ii) Let $g(x)=\frac{1}{x}$, then $\int_{a}^{\infty} \frac{1}{x} d x$ is divergent, for $R(x)<\frac{1}{x}=R_{g}(x)$, from Theorem 2.2, we have that $\int_{a}^{+\infty} f(x) d x$ is divergent.

Combining [3], we have that $\int_{a}^{+\infty} f(x) d x$ is possible convergent or divergent if $\lim _{x \rightarrow+\infty} x R(x)=1$ from some possible examples. In fact, we can continue to study convergence as follow:

Theorem 2.4 Assume that $f(x)$ is non-negative differentiable function in interval $[a,+\infty)$ and

$$
\lim _{x \rightarrow+\infty} x R(x)=1 \text {. }
$$

(i) If $\lim _{x \rightarrow+\infty} \ln x[x R(x)-1]>1$, then $\int_{a}^{+\infty} f(x) d x$ is convergent,

(ii) If $\lim _{x \rightarrow+\infty} \ln x[x R(x)-1] \leq 1$, then $\int_{a}^{+\infty} f(x) d x$ is divergent.

Proof: (i) We can obtain that $R(x)$ is monotone-nonincreasing function, for all $\forall k>0$, we have

$$
\begin{aligned}
& f(x) \leq c_{0} \exp \{-x R(x)\} \leq \\
& c_{0} \exp \left\{-\left(1+\frac{1+k}{\ln x}\right)\right\}< \\
& c_{0} \exp \left\{-\frac{1+k}{\ln x}\right\}=\frac{c_{0}}{x^{1+k}}
\end{aligned}
$$

From Ordinary Comparison Test we can obtain that

$$
\int_{a}^{+\infty} f(x) d x<+\infty
$$


(ii)Method1: Let $R(x) \leq \frac{1+\ln x}{x \ln x}$, we have that $-\ln f(x) \leq \ln (x \ln x)$, i.e. $f(x) \geq \frac{1}{x \ln x}$, by $\int_{a}^{\infty} \frac{1}{x \ln x} d x$ is divergent, combining Ordinary Comparison test, we can obtain that $\int_{a}^{+\infty} f(x) d x$ is divergent.

Method2: Using the condition, we obtain that

$$
R(x) \leq \frac{1+\ln x}{x \ln x}=R_{g}(x)
$$

Where $g(x)=\frac{1}{x \ln x}$, and $\int_{a}^{\infty} \frac{1}{x \ln x} d x$ is divergent. From theorem2.2, we have that $\int_{a}^{+\infty} f(x) d x$ is divergent too.

Using the conclusions above, we can easily think of the following examples.

Example 1: Discuss the convergence of $\int_{2}^{\infty} \frac{1}{x^{p} \ln ^{q} x} d x$.

Solution: For its exponential integrating factor

$$
R(x)=-\frac{p+c q x^{c}}{x},
$$

We easy to know that $\lim _{x \rightarrow+\infty} x \frac{p \ln x+q}{x \ln x}=p$, using theorem 2.3, we have that the integration is convergent if $p>1$, and is divergent if $p<1$; when $p=1$, we easy to know $x[R(x)-1]=q$, using theorem 2.4, we get that the integration is convergent when $q>1$; the integration is divergent when $q \leq 1$.

Example2: Discuss the convergence of

$$
\int_{1}^{\infty} x^{p} \exp \left(q x^{c}\right) d x
$$

Solution: For its exponential integrating factor is

$$
R(x)=-\frac{p+c q x^{c}}{x}
$$

We easy to know that $\lim _{x \rightarrow+\infty} x R(x)=-\left[p+c q x^{c}\right]$, using theorm2.3, we have $\int_{1}^{\infty} x^{p} \exp \left(q x^{c}\right) d x$ is convergence if $\lim _{x \rightarrow+\infty} p+c q x^{c}<-1$, i.e. $\quad c \leq 0$ or $q=0, p<-1$ or $\mathrm{c}>0, \mathrm{q}<0$.

\section{CONSTRUCTING CONVERGENT INFINITE INTEGRAL AND POSSIBLE APPROACH}

From Theorem 2.4, we can obtain that the convergence and divergence of $\int_{a}^{+\infty} f(x) d x$ is determined by the exponential integrating factor of $f(x)$.

Suppose that $\int_{a}^{+\infty} f(x) d x$ and $\int_{a}^{+\infty} f(x) g(x) d x$ are convergent, we give the following conditions of the nonnegative function $g(x)$.

Using the exponential integrating factor of $f(x) g(x)$,

i.e. $R_{f g}(x)=R_{f}(x)+R_{g}(x)$,

if $\lim _{x \rightarrow+\infty}\left(x\left[R_{f}(x)+R_{g}(x)\right]\right)>1$, then

$\int_{a}^{+\infty} f(x) g(x) d x$ is convergent. In fact, we can construct some convergent infinite integral for some specific needs.

Theorem 3.1 Assume that $\int_{a}^{+\infty} f(x) d x$ is convergent, if the non-negative function $g(x)$ is monotonous and bounded, then $\int_{a}^{+\infty} f(x) g(x) d x$ is convergent.

Proof: Combining the conditions and theorem 2.4, we know that $\lim _{x \rightarrow+\infty} \ln x[x R(x)-1]>1$. If $g(x)$ is decreasing and lower bounded,

Then $R_{g}(x)>0$ and $R_{f g}(x)=R_{f}(x)+R_{g}(x)$ hold. Obviously, $\lim _{x \rightarrow+\infty}\left(x\left[R_{f g}(x)-1\right]\right)>1$; If $g(x)$ is increasing and upper bounded, then $\int_{a}^{x} R_{g}(t) d t$ is convergent, i.e. $\lim _{x \rightarrow+\infty} x \ln x R_{g}(x)=0$, such that

$$
\lim _{x \rightarrow+\infty} \ln x\left[x R_{f g}(x)-1\right]=\lim _{x \rightarrow+\infty} \ln x\left[x R_{f}(x)-1\right]>1 .
$$

From all above, we can obtain that $\int_{a}^{+\infty} f(x) g(x) d x$ is convergent.

Theorem 3.2 Suppose $\int_{a}^{+\infty} f(x) d x$ is convergent where $f(x)$ is continuous and nonnegative in $[a,+\infty)$.

(i)If $\lambda<\infty$ and $\alpha<\lambda-1$, then $\int_{a}^{\infty} x^{\alpha} f(x) d x$ is convergent.

(ii)If $\lambda=\infty, \forall \alpha \in R$, then $\int_{a}^{\infty} x^{\alpha} f(x) d x$ is convergent, 
(iii)If $\lambda=\infty$ and $\beta<\lim _{x \rightarrow+\infty} \frac{\ln \left[\int_{1}^{x}\left[R(t)-\frac{1}{t}\right] d t+1\right]}{\ln x}$, Then $\int_{a}^{\infty} \exp \left\{x^{\beta}\right\} f(x) d x$ is convergent, where $\lambda=\lim _{x \rightarrow+\infty} \inf x R(x)$,

Proof: (i) For $R(x)-\frac{\alpha}{x}$ is the factor function of $x^{\alpha} f(x)$, if $\alpha<\lambda-1$, then $\lim _{x \rightarrow+\infty} x R(x)-\alpha>1$,i.e.

$$
\lim _{x \rightarrow+\infty} x\left[R(x)-\frac{\alpha}{x}\right]>1
$$

Using theorem 2.3, we get $\int_{a}^{\infty} x^{\alpha} f(x) d x$ is convergent.

(ii) Easy to get by (i).

(iii) From condition $x^{\beta}<\int_{1}^{x}\left[R(t)-\frac{1}{t}\right] d t+1$, i.e.

$$
\int_{1}^{x} \beta t^{\beta-1} d t<\int_{1}^{x}\left[R(t)-\frac{1}{t}\right] d t
$$

We have $\beta x^{\beta-1}<R(x)-\frac{1}{x}$, from $\exp \left\{x^{\beta}\right\} f(x)$, we obtain its factor function $R(x)-\beta x^{\beta-1}$, using theorm2.3, we get $\lim _{x \rightarrow+\infty} x\left[R(x)-\beta x^{\beta-1}\right]>1$. Base on L'Hospital rule, we have

$$
\beta<\lim _{x \rightarrow+\infty} \frac{x R(x)-1}{\int_{1}^{x}\left[R(t)-\frac{1}{t}\right] d t+1} .
$$

Thus, we have that $\int_{a}^{\infty} \exp \left\{x^{\beta}\right\} f(x) d x$ is convergent.

\section{Conclusions And Prospect}

In this paper, we get a new test method for the convergence and divergence of infinite integral, as everyone knows, defect integral can be changed into infinite integral by $x=\frac{1}{t}$ so we can expands our method on the defect integral.

We can almost judge the convergence of the infinite integrals by the conclusions refer to in Section 2. So we discuss some questions of infinite integral by the properties of the convergent infinite integral. In fact, the most important application in Section 3 is to construct plenty of distributions in the probability field especially in the Risk Theory. The claim distribution is vital for both the insured and the insurance company [4-7], constructing suitable distributions is beneficial to need for the insured or development of the insurance companies. This is a topic for future research.

\section{ACKNOWLEDGMENT}

The authors wish to give their sincere thanks to the anonymous referees for their valuable suggestions and helpful comments which improved the presentation of the paper.

\section{REFERENCES}

[1] East China normal university, Mathematical analysis [M], Higher education press, 2001. (In Chinese)

[2] Wen Z.Y., Convergence of the infinite integrals of a new identification method [J], Mathematics,2005.21(2):111-112. (In Chinese)

[3] Luo L.P., The discussion of the necessary conditions for infinite integral convergence [J], Advanced mathematics research, 2005,8(4):19-21. (In Chinese)

[4] Embrechts P, Veraverbeke N. Estimates for the propabilty of ruin with special emphasis on the possibility of large claims. Insurancee Math Econ, 1982, 1: 55-72

[5] Klüppelberg C., Subexponential distributions and characterizations of relateed class. Probab. Th. Rel.Fields,1989, 82: 259-269.

[6] Cline D B H. Convolutions of distributions with exponential and subexponential tails. Austral Math Soc (Series A), 1987, 43: 347-365

[7] Yin Chuancun. A local theorem for the probability of ruin. Science in China (Series A). 2004, 47: 711-721. 